\title{
Quarkonia dynamics in the QGP with open quantum sys- tems
}

\author{
Stéphane Delorme ${ }^{1, *}$, Thierry Gousset ${ }^{1}$, Roland $\mathrm{Katz}^{1}$, and Pol-Bernard Gossiaux ${ }^{1}$ \\ ${ }^{1}$ SUBATECH, Université de Nantes, IMT Atlantique, IN2P3/CNRS, 4 rue Alfred Kastler, 44307 Nantes \\ cedex 3, France
}

\begin{abstract}
We investigate the real-time dynamics of a correlated heavy quarkantiquark pair inside the Quark-Gluon Plasma using new quantum master equations derived from first QCD principles and based on the work of Blaizot \& Escobedo [4]. The full equations are directly numerically solved in one-dimension to reduce computing costs and is used to gain insight on the dynamics in both a static and evolving medium following a Björken-like temperature evolution. The effect of the initial state on the dynamics is also studied.
\end{abstract}

\section{Quarkonium suppression and recombination}

A key observable for the study of the Quark-Gluon Plasma (QGP) in nucleus-nucleus (AA) collisions is the suppression of quarkonia. It is characterized by a lower production of quarkonia in A-A collisions compared to proton-proton ( $\mathrm{p}-\mathrm{p}$ ) collisions. To explain quarkonia suppression, several effects are at play. Due to the presence of the QGP, the interaction between the heavy quark and antiquark composing a quarkonium is modified, as numerous other color charges are present. The density of color charges also increases as temperature increases, leading to a stronger screening of the $Q \bar{Q}$ interaction and a melting of the pairs. [1] The bound $Q \bar{Q}$ pair may also scatter on the plasma constituents (light quarks and gluons), which can also lead to the dissociation of the pair. Those two effects are usually described by a complex potential, which depends on the medium temperature and the relative distance between the two quarks. The screening effects are described by the real part V of this potential and the dynamical effects are described by the imaginary part $\mathrm{W}$ of this potential. The combination of both screening and collisions leads to quarkonia suppression. This picture is however incomplete: one would expect a stronger suppression as the collision energy increases, however, experimental measurements at high-energy colliders (such as the LHC and the RHIC) showed a more complicated pattern. Indeed, suppression was found to be much smaller than expected for the $J / \Psi$ with respect to some quantities [3]. A possible cause for this smaller suppression is the recombination of uncorrelated charm quarks and antiquarks during the evolution and the hadronization phase. Recombination is therefore crucial for charmonia phenomenology, as the number of $c$ and $\bar{c}$ produced in A-A collisions is very high. In recent years, a lot of effort has been done to provide a geniune quantum treatment of the real-time dynamics of heavy quarkonia in the QGP, using the open quantum systems formalism. In that framework, one can study the dynamics of a quantum system (a quarkonium) in interaction with an environment (the QGP). As we are only interested in the $Q \bar{Q}$

\footnotetext{
*e-mail: delorme@ subatech.in2p3.fr
} 
pair dynamics, we perform a trace on the environment degrees of freedom in order to obtain a quantum master equation describing the quarkonium real-time dynamics.

\section{The model}

This work is based on the previous work by Blaizot \& Escobedo [4]. In their article, they derived coupled quantum master equations in the quantum brownian motion regime (which is valid for temperatures $T>>200 \mathrm{MeV}$ ) by assuming a weak coupling between the plasma constituants (light quarks and gluons) and the heavy quarks forming the pair. Those equations take into account color degrees of freedom and can be written as:

$$
\frac{d}{d t}\left(\begin{array}{l}
\mathcal{D}_{s} \\
\mathcal{D}_{o}
\end{array}\right)=\mathcal{L}\left(\begin{array}{l}
\mathcal{D}_{s}\left(\mathbf{s}, \mathbf{s}^{\prime}, t\right) \\
\mathcal{D}_{o}\left(\mathbf{s}, \mathbf{s}^{\prime}, t\right)
\end{array}\right),
$$

where $\mathcal{D}_{s}$ and $\mathcal{D}_{o}$ are respectively the singlet and octet density matrices. $\mathbf{s}$ and $\mathbf{s}^{\prime}$ are respectively the relative distance between the quark and the antiquark and the conjugated variable. More specifically, one can decompose the $\mathcal{L}$ operator in the following way:

$$
\mathcal{L}=\left(\begin{array}{ll}
\mathcal{L}_{s s} & \mathcal{L}_{s o} \\
\mathcal{L}_{o s} & \mathcal{L}_{o o}
\end{array}\right),
$$

with the various $\mathcal{L}_{s s}, \mathcal{L}_{s o}, \mathcal{L}_{o s}$ and $\mathcal{L}_{o o}$ operators describing transitions between color states. Another way of decomposing $\mathcal{L}$ (and the other color transition operators) is to write $\mathcal{L}=$ $\mathcal{L}_{0}+\mathcal{L}_{1}+\mathcal{L}_{2}+\mathcal{L}_{3} . \mathcal{L}_{0}$ describes the free motion of the pair, $\mathcal{L}_{1}$ involves the real part of the imaginary potential $\mathrm{V}$ and describes the static screening effects, $\mathcal{L}_{2}$ is related to the fluctuations in the medium which involves the imaginary part of the potential W) and $\mathcal{L}_{3}$ describes dissipative effects in the medium, which involves derivatives of W. A key property of well-defined quantum master equations is the conservation over time of the density operator positivity. In their approach, the equations derived by Blaizot \& Escobedo break the positivity at the $\mathcal{L}_{3}$ level. In our work, we extended their equations ${ }^{1}$, resulting in the modification of $\mathcal{L}_{3}$ operator and the presence of a new higher-order operator $\mathcal{L}_{4}$. With the new equations, positivity is preserved and the master equation is well-defined. We chose to solve the quantum master equations numerically in a one-dimensional case, in order to reduce the computing cost, as it is sufficient to gain insight on the dynamics. The resolution was performed on a bi-dimensional grid of size $20 \mathrm{fm} \times 20 \mathrm{fm}$, with a spatial step $\Delta s=0.04 \mathrm{fm}$ and a time step $\Delta t=0.1 \mathrm{fm} / \mathrm{c}$, using the Crank-Nicolson scheme.

\section{One-dimensionnal dynamics of a quarkonium in the QGP}

\subsection{Color dynamics}

In this section, we consider a QGP with a fixed temperature, set to $T=300 \mathrm{MeV}$ and consider two cases: 1) a "QED-like" case where color transitions are forbidden and 2) a proper QCD case where color transitions are taken into account. In both cases, we take as initial state the 1S-like eigenstate, obtained from the real part $\mathrm{V}$ of the vacuum potential. We then perform at each time the instantaneous projections on the first three eigenstates (as if the freeze-out was instantaneous), defined as $w_{n}^{Q E D}=\left\langle\Psi_{n}^{Q E D}|\mathcal{D}| \Psi_{n}^{Q E D}\right\rangle$ in the QED-like case and $w_{n}^{Q C D}=\left\langle\Psi_{n}^{Q C D}\left|\mathcal{D}_{s}\right| \Psi_{n}^{Q C D}\right\rangle$ in the QCD case. Figure 1 shows the evolution over time of

\footnotetext{
${ }^{1}$ Following a procedure described in Appendix B of [4].
} 
the projections on the eigenstates. One can clearly see that the populations of states in the QCD case are smaller than in the QED-like case. This can be explained by the color transitions, which transfer part of the norm to octet color sector. Color transitions are therefore crucial to understand properly the dynamics of a quarkonium in the Quark-Gluon Plasma.

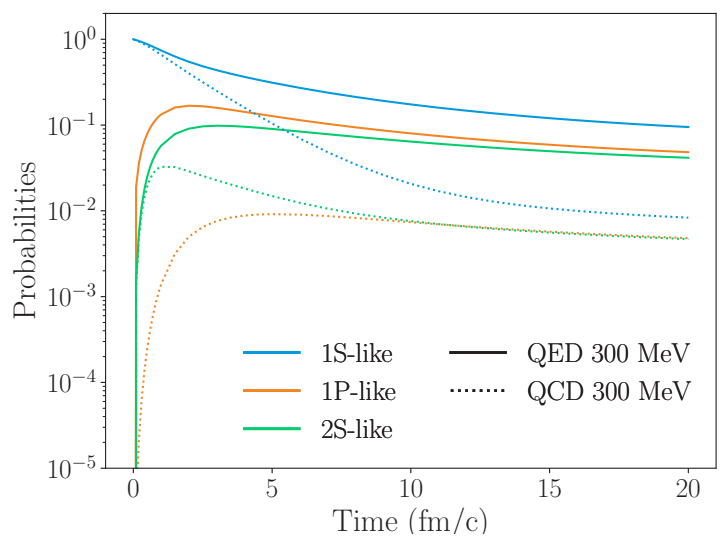

Figure 1. Evolution over time of the vacuum eigenstates populations for a temperature $T=300 \mathrm{MeV}$ with color transitions (dashed lines) and without color transitions (solid lines)

\subsection{Fixed temperature case}

We first consider a medium with fixed temperature and take as the initial state the 1S-like eigenstate obtained from the vacuum potential V. We show on figure 2 the evolution over time of the populations of the first three eigenstates of the vacuum potential for three different medium temperatures $T=200,300$ and $400 \mathrm{MeV}$.
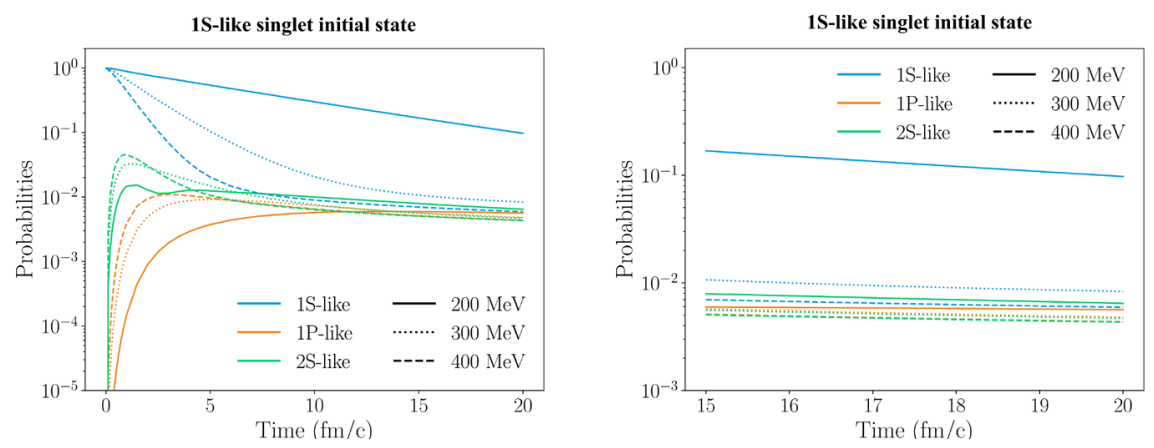

Figure 2. Left panel: Evolution over time of the vacuum eigenstates populations for a temperature $T=200,300$ and $400 \mathrm{MeV}$. Right panel: Zoom on the late-time evolution.

We can observe at early times a transient phase where transitions from the initial 1Slike state occur, followed by an equilibration phase of the populations. We can note that the $2 \mathrm{~S}$-like state is populated faster than the $1 \mathrm{P}$-like state, due to the different nature of the 
transitions. In the case of the $2 \mathrm{~S}$-like state, a direct transition from the $1 \mathrm{~S}$-like state (due to the real part of the potential V) is possible, while for the 1P-like state, dipolar transitions must occur. Those transitions however also induce a color state transition, meaning that the transition from a $1 \mathrm{~S}$-like singlet state to a 1P-like singlet state requires multiple dipolar transitions, resulting in a slower population of the $1 \mathrm{P}$-like state. At later times, the decay rate is the same for all three eigenstates (except for $T=200 \mathrm{MeV}$ ) and populations seems to follow a temperature distribution. However, they do not follow a Maxwell-Boltzmann distribution at the considered temperatures. The evolution of the populations is characterized by the interplay between binding, diffusion and transition between states.

$$
\mathcal{D}_{s} \mathbf{T}=300 \mathrm{MeV} \quad \text { initial 1S-like singlet }
$$
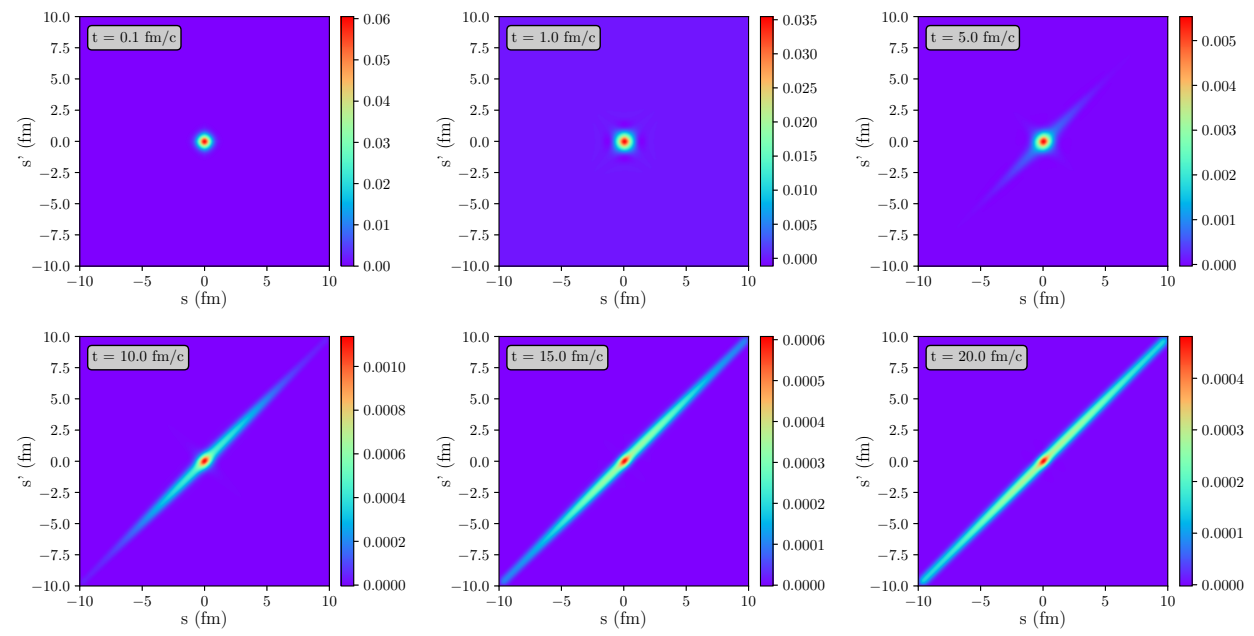

Figure 3. Evolution of the singlet density matrix $\mathcal{D}_{s}$ over time for a temperature $T=300 \mathrm{MeV}$.

Figure 3 shows the evolution of the singlet density matrix $\mathcal{D}_{s}$ over time. The initial state progressively delocalizes along the $s=s^{\prime}$ axis and two components are observed: 1 ) a remnant of the initial state, present around $s=s^{\prime}=0 \mathrm{fm}$ and 2) a semi-classical-like component on the rest of the $s=s^{\prime}$ axis. We can observe that the width of the distribution along the $s=-s^{\prime}$ is small, indicating that the quarkonium evolution is affected by strong decoherence effects induced by the QGP.

We now change the initial state and take a P-like state in the octet color channel. The evolution over time of the state populations for that initial state is shown in Figure 4. We can observe that compared to the singlet initial state, the final populations are greater, due to the transitions from all possible octet channels. The second observation we can make is the formation of bound states due to color transitions at early times. One should however note that the two quarks are already very close to each other at the start of the evolution, which helps the early time formation. 


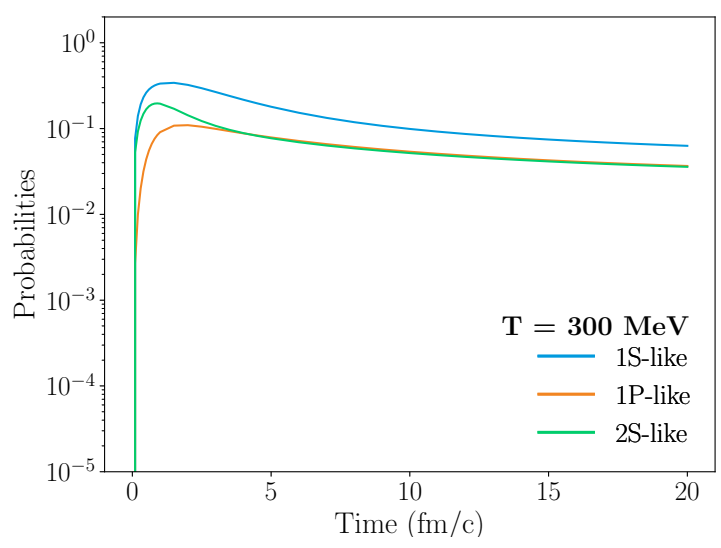

Figure 4. Evolution over time of the vacuum eigenstates populations for a temperature $T=300 \mathrm{MeV}$ with an initial P-like octet state.

The evolution of the singlet density matrix $\mathcal{D}_{s}$ over time is shown in figure 5 . We can see that the singlet state formed at early times from color transitions also delocalize progressively along the $s=s^{\prime}$ axis. The same observations as in the color singlet initial state case can be made, indicating that the limit reached at later times may be independent of the initial state considered.

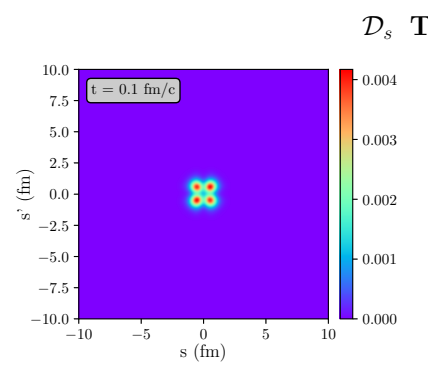

T $=300 \mathrm{MeV} \quad$ initial P-like octet
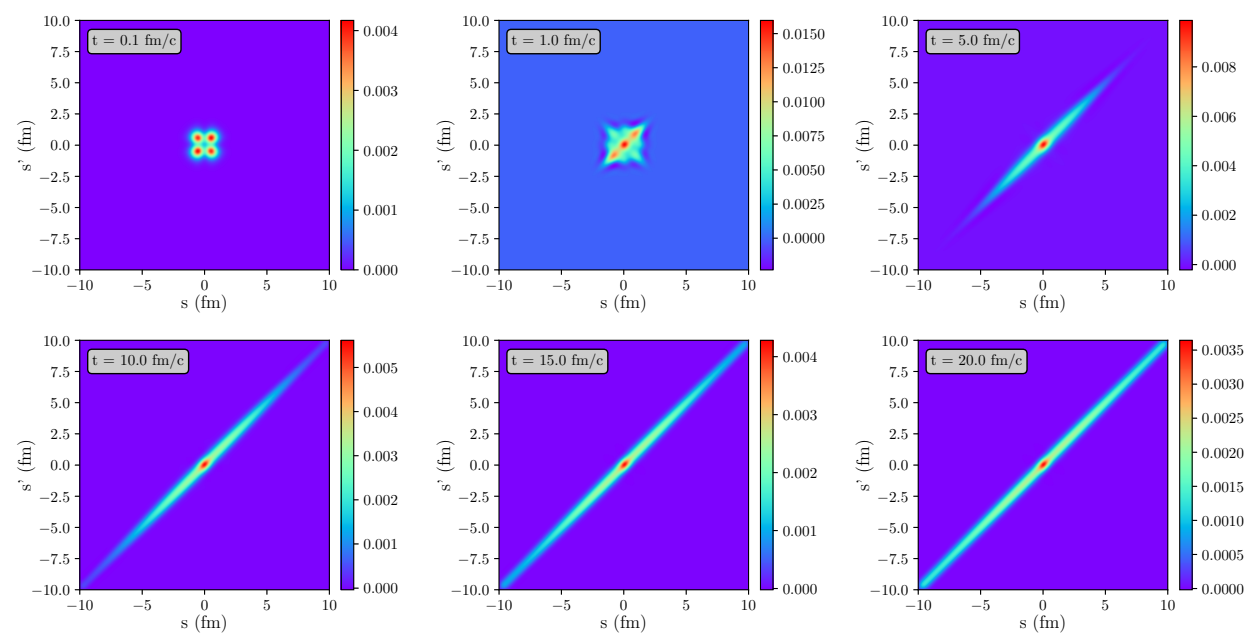

Figure 5. Evolution of the singlet density matrix $\mathcal{D}_{s}$ over time for a temperature $T=300 \mathrm{MeV}$ and an octet P-like initial state. 


\subsection{Cooling medium case}

We now relax the fixed temperature medium approximation used previously and adopt a medium temperature evolution following Björken's model [5], $T(t)=T_{0}\left(\frac{t_{0}}{t_{0}+t}\right)^{1 / 3}$, where $T_{0}$ is the medium initial temperature and $t_{0}$ the initial time taken to be equal to $1 \mathrm{fm} / \mathrm{c}$. We consider a QGP medium with initial temperature $T_{0}=600 \mathrm{MeV}$ and an initial P-like state in the octet color channel. We perform as before the instantaneous projection of the singlet density matrix $\mathcal{D}_{s}$ on the vacuum eigenstates. The results obtained are shown in figure 6 . We again observe the creation at early times of excited singlet bound states and the progressive re-equilibration of the populations, followed by a decay phase. One can also note that starting from $t \sim 13 \mathrm{fm} / \mathrm{c}$, the $1 \mathrm{~S}$-like eigenstate starts to get repopulated. This can be explained by the progressive cooling of the medium, leading to the binding of excited $c \bar{c}$ pairs and $c \bar{c}$ pairs from the continuum. This effect was not present when the medium temperature was fixed, indicating that a realistic treatment of the medium is required.

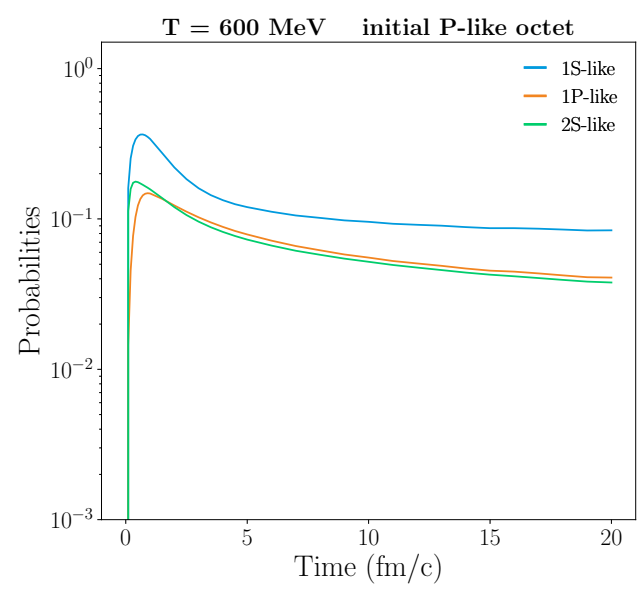

Figure 6. Evolution over time of the vacuum eigenstates populations from an initial temperature $T_{0}=$ $600 \mathrm{MeV}$.

\section{Conclusion}

We presented results on real-time quarkonium dynamics in the quark-gluon plasma described by new coupled quantum master equations. The equations involve transitions between color states as well as dissipative effects, and were solved numerically in one dimension. The global evolution is the result of the interplay between binding effects, diffusive effects and transitions between states. The analysis of the density matrix showed the delocalization of the initial state along a specific direction in the $\left(s, s^{\prime}\right)$ space and exhibited a semi-classical-like behaviour. The analysis of this semi-classical component is under way and updated results will be published in the near future.

\section{Acknowledgements}

The authors thank Jean-Paul Blaizot and Alexander Rothkopf for valuable discussions. 


\section{References}

[1] T. Matsui, H. Satz, Phys. Lett. B 178, 416 (1986)

[2] D. Lafferty, A. Rothkopf, Phys. Rev. D, 101 (2020)

[3] ALICE collaboration, Phys. Letter. B., 766, 212-224 (2017)

[4] J.P. Blaizot, M.A. Escobedo, J. High Energ Phys. 2018, 34 (2018)

[5] J.D. Björken, Phys. Rev. D, 27, 140-151 (1983) 